\title{
Topical issue: 13th international symposium on high pressure low temperature plasma chemistry (HAKONE XIII)
}

The 13th international symposium on high pressure, low temperature plasma chemistry (HAKONE XIII) held in Kazimierz Dolny, Poland from September 9 to 14, 2012 was organized by the Institute of Electrical Engineering and Electrotechnologies, Lublin University of Technology LUT, the Commission of Low-Temperature Plasma Chemistry of Polish Academy of Science PAN - Branch in Lublin, the Association of Polish Electrical Engineers (SEP) and the Polish Society of Theoretical and Applied Electrical Engineering (PTETiS).

This specialized biannual conference derives its name from the small resort area Hakone in Japan where it was first organized 25 years ago, in 1987. It is purposely kept small with no parallel sessions and preferentially meets in remote locations to stimulate scientific discussion. This year we returned to the location where the late Professor Iwo Pollo from Lublin Technical University, one of the HAKONE initiators, had organized HAKONE II in 1989. This year HAKONE symposium gathered 85 participants from 16 countries. 65 oral and poster presentations of HAKONE XIII are available in the Book of Contributed Papers and on the USB stick distributed to all participants of the symposium. More details about the HAKONE XIII symposium, scientific program and Book of Contributed Papers can be found in: http://ipee.pollub.pl/hakone/HAKONE_XIII/Information.html/

In the scientific program of the symposium, besides the topics dealing with fundamental problems and diagnostics of high pressure low temperature plasmas (HPLTPs), the homogenous DBD arrangements, microplasma devices, pulsed coronas, gliding arc discharges and atmospheric-pressure plasma jets, as well as fields of their environmental and biomedical applications were represented.

Traditionally, the International Scientific and Organisation Committee (ISOC) has invited lecturers to give talks presenting progress in HPLTPs generation and application. Six invited talks covered the following topics:

- biomedical applications of cold plasmas by Alexander Fridman (USA),

- proton transfer mass spectrometry fundamentals, advances and applications, by Tilman Märk (Austria),

- photoionization/streamer propagation by Sander Nijdam (Netherland),

- progress in industrial ozone generation by Jose Lopez (USA),

- decomposition of perfluoro compounds in water using discharge by Kouichi Yasuoka (Japan),

- plasma for saving energy, health and environment by Zbigniew Kołaciński (Poland).

The Hakone ISOC together with editorial board of the European Physical Journal - Applied Physics prepared a special issue of the journal devoted to the symposium. The ISOC members preselected papers for publication in the journal and 25 of them which successfully passed the peer review procedure are presented in this issue.

The next meeting, HAKONE XIV, will be organized in 2014 at Zinnowitz on the shore of the Baltic sea by the University of Greifswald and the Leibniz Institute for Plasma Science and Technology (INP).

We would like to use this opportunity to invite the readers of this journal to the next HAKONE symposium.

Henryka Danuta Stryczewska,

Local Organizing Committee

Ulrich Kogelschatz,

International Scientific and Organizing Committee 